\title{
Light regulation of pigment and photosystem biosynthesis in cyanobacteria
}

Ming-Yang $\mathrm{Ho}^{1,2, *}$ Nathan $\mathrm{T}$ Soulier ${ }^{1, *}$, Daniel $\mathrm{P}$ Canniffe ${ }^{1}$,
Gaozhong Shen ${ }^{1}$ and Donald A Bryant ${ }^{1,2,3}$

\section{Addresses:}

${ }^{1}$ Department of Biochemistry and Molecular Biology, The Pennsylvania State University, University Park, PA 16802, USA

2 Intercollege Graduate Degree Program in Plant Biology, The Pennsylvania State University, University Park, PA 16802, USA

${ }^{3}$ Department of Chemistry and Biochemistry, Montana State University, Bozeman, MT 59717, USA

*These authors contributed equally to this review.

Corresponding author: Bryant, Donald A (dab14@psu.edu) 


\begin{abstract}
:
Most cyanobacteria are obligate oxygenic photoautotrophs, and thus their growth and survival is highly dependent on effective utilization of incident light. Cyanobacteria have evolved a diverse set of phytochromes and cyanobacteriochromes (CBCRs) that allow cells to respond to light in the range from $\sim 300 \mathrm{~nm}$ to $\sim 750 \mathrm{~nm}$. Together with associated response regulators, these photosensory proteins control many aspects of cyanobacterial physiology and metabolism. These include far-red light photoacclimation (FaRLiP), complementary chromatic acclimation (CCA), low-light photoacclimation (LoLiP), photosystem content and stoichiometry (long-term adaptation), short-term acclimation (state transitions), circadian rhythm, phototaxis, photomorphogenesis/development, and cellular aggregation. This minireview highlights some discoveries concerning phytochromes and CBCRs as well as two acclimation processes that improve light harvesting and energy conversion under specific irradiance conditions: FaRLiP and CCA. (123 words)
\end{abstract}




\section{Introduction}

Members of the phylum Cyanobacteria are unique among prokaryotic chlorophototrophs (i.e., chlorophyll-based phototrophs): nearly all are oxygen-evolving, obligate photoautotrophs, and they often fix dinitrogen as well. The cyanobacterial photosynthetic apparatus comprises three principal energy transducing and light-harvesting complexes: Photosystem I (PSI; plastocyanin/cytochrome $c_{6}$ : ferredoxin photooxidoreductase) [1,2]; Photosystem II (PSII, water:plastoquinone photooxidoreductase) [3]; and phycobilisomes (PBS) [4]. PSII and PSI are interconnected by electron shuttles plastoquinone and cytochrome $c_{6}$ (or plastocyanin) and the cytochrome $b_{6} f$ complex, which acts as a plastoquinol:cytochrome $c_{6} /$ plastocyanin oxidoreductase [5]. Acting together, these complexes allow cells to photooxidze water and reduce ferredoxin (and ultimately $\mathrm{NADP}^{+}$via ferredoxin:NADP ${ }^{+}$oxidoreductase) [6]. The proton gradient produced by this electron transport chain is used by ATP synthase to generate ATP for carbon fixation and cell growth [7].

As might be expected for organisms whose existence depends upon light, cyanobacteria have evolved a complex set of photoreceptors that control many aspects of their physiology and metabolism. For example, the genome of Fremyella diplosiphon PCC 7601, also known as Tolypothrix tenuis PCC 7601, encodes 27 different phytochrome or cyanobacteriochrome (CBCR) photoreceptors, some of which are predicted to contain multiple light-sensing domains [8]. Similarly, the genome of Nostoc punctiforme PCC 73102 encodes more than 30 putative photoreceptors; 21 of these proteins have been shown to bind bilin chromphores $[9,10]$. A recent comparative genomic analysis of 44 cyanobacterial genomes showed that, in general, the number 
of photoreceptors encoded within cyanobacterial genomes increases with genome size and with developmental complexity [10].

By shuffling and combining different protein domains, chromophore types, the number of covalent linkages between cysteines and the chromophore, and the chromophore environment, evolution has created proteins with a remarkable capacity to sense light wavelengths (colors) from $300 \mathrm{~nm}$ to $>740 \mathrm{~nm}$ [11-14], which can influence cellular physiology and metabolism by coupling to various output domains (see Figure S1 in [9]). In general, cyanobacteria can sense ten different wavelength ranges (light colors): near-ultraviolet (NUV, 300-395 nm), violet (VL, 395-410 nm), blue (BL, 410-485 nm), teal (TL, 485-515 nm), green (GL, 515-570 nm), yellow (YL, 570-590 nm), orange (OL, 590-615 nm), red (RL, 615-675 nm), far-red (FRL, 675-740 $\mathrm{nm}$ ), and near-infrared (NIR, $>740 \mathrm{~nm})$ [11-14]. This diversity of photosensors and mechanisms of light perception is matched by the variety of physiological responses that cells exhibit in response to light. As summarized in Figure 1 and as discussed below, these may include but are not limited to Far-ㅌed Light Photoacclimation (FaRLiP), Complementary Chromatic Acclimation (CCA), Low-Light Photoacclimation (LoLiP), photosystem content and stoichiometry, short-term acclimation (state transitions), circadian rhythm, phototaxis, photomorphogenesis/development, and cellular aggregation. This minireview will highlight recent developments in defining the roles of light in the sensing and control of some of these processes that affect pigment biosynthesis and the photosynthetic apparatus.

\section{Phytochrome and cyanobacteriochrome photosensors: modular sensor design leads to remarkable diversity for sensing and responding to light}


Phytochromes are photoreceptors that use cysteinyl thioether-linked, bilin chromophores to regulate biological responses to RL/FRL. Phytochromes characteristically contain PAS

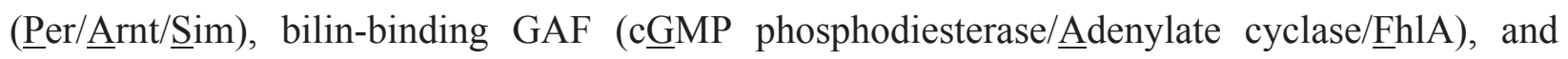
PHY (Phytochrome) domains. These domains occur in a conserved, knotted PAS-GAF-PHY photosensory module, in which phycocyanobilin, is usually bound to a conserved cysteine residue in the GAF domain [11,15-18]. Cyanobacteria also produce knotless phytochromes, in which a GAF-PHY photosensory module senses RL/FRL (e.g., RfpA) [17-19]. In either case, isomerization of the 15,16 double bond of the chromophore photoconverts a RL-absorbing, $15 Z$ $\left(\mathrm{P}_{\mathrm{r}}\right)$ form into a FRL-absorbing, $15 E\left(\mathrm{P}_{\mathrm{fr}}\right)$ form (Figure 2). Reversion of the metastable $\mathrm{P}_{\mathrm{fr}}$ state can also occur thermally in the dark. In general, proteins with chromophores that exhibit slow dark reversion rates and faster photoconversion rates are suitable for sensing different light wavelengths/colors. Conversely, proteins that exhibit rapid dark reversion rates probably function as power sensors and are best suited to sense light quantity/irradiance [20].

The largest and most diverse group of cyanobacterial photoreceptors are the CBCRs, which extend the range of light wavelengths perceived to shorter wavelengths of visible light or even NUV [11,18]. At least eight subfamilies of CBCRs are known, many of which contain a second cysteine motif (e.g., DXCF, DXCIP, insert-Cys, etc.) in the GAF domain; this allows the formation of a thioether linkage to position C-10 of the chromophore (see Figure 2) $\left[12,15,21^{\circ}\right.$ 25"]. Unlike most phytochromes, the GAF domains of CBCRs are usually able to bind a chromophore and are photoactive in the absence of PAS and/or PHY domains [11,25"]. CBCR diversity is partly achieved by varying the bound bilin, which can be phycocyanobilin, phycoviolobilin or biliverdin. Diverse spectral-tuning mechanisms, including dual-cysteinyl thioether linkages and specific arrangements of aromatic residues surrounding the chromophore 
[9,11-25"], further enhance photosensor diversity. Photosensors of FRL or NIR can be associated with a biliverdin chromophore instead of phycocyanobilin $[14,18]$. The blue/green photocycles in some DXCF CBCRs are due to reversible thioether bond formation to carbon C-10 of the chromophore (Figure 2), and in some GAF domains, phycoviolobilin or mixtures of this chromophore and phycocyanobilin occur $\left[9,15,21^{\circ}, 24,25^{*}\right]$. Recent studies of CBCR proteins, which have GAF domains lacking the canonical cysteine demonstrated that a second cysteine ligates an unidentified chromophore that unidirectionally photoconverts in response to GL [21"]. Protonation of the isomerized chromophore can also play an important role in defining the spectroscopic properties of CBCRs $\left[26^{\circ}\right]$. These sensory elements are most commonly associated with phosphoacceptor/histidine kinase output domains, although methyl-accepting chemotaxis, GGDEF (cyclic-di-GMP synthase), and some other types of output domains also occur $[9,15,16]$. When compared across different cyanobacteria, similar light-sensing modules can be coupled to different output domains [9-11,16].

The remarkable diversity of phytochrome-like and $\mathrm{CBCR}$ photoreceptors allows organisms to regulate many physiological processes in response to the incident light. For example, N. punctiforme PCC 73102 has 21 phytochrome and CBCR photoreceptors that collectively contain 41 biochemically validated, bilin-binding GAF domains [9]. This indicates that many of these proteins are able to integrate information about multiple wavelengths of light to formulate a more precise output signal. In one extreme example in photoreceptor NpF2164, seven GAF domains, six of which bind phycocyanobilin covalently, are fused to a methylaccepting chemotaxis protein output domain. Two of the GAF domains contain a DXCF motif containing an additional cysteine residue that forms a second covalent linkage to C-10 of phycocyanobilin $\left[12,15,22-25^{*}\right]$. This reduces the number of conjugated double bonds in the 
chromophore and produces domains that can respond to shorter wavelengths of light $\left[21^{\circ}, 24\right]$. Collectively, the six GAF domains in NpF2164 allow this protein to integrate information about incident radiation over the range from $\sim 300$ to $700 \mathrm{~nm}$-nearly the entire spectral region covered by all CBCR photosensors.

These flexible changes, coupled to different types of output modules, allow cells to sense light and control diverse biological processes (Figure 1) $\left[9,11,16-19^{*}, 27-30\right]$. In the following sections, we describe some recent advances in defining the roles of phytochromes and CBCRs in light control of photosynthesis in cyanobacteria (Figure 1). We will principally focus on two processes, FaRLiP and CCA. Other processes, including LoLiP, circadian rhythm, long-term adaptation (photosystem content and ratio) and short-term acclimation (state transitions) to light intensity will not be discussed in detail. It should be noted that not all light-regulated processes affect the photosynthetic apparatus. For example, CBCRs can also regulate phototaxis $[11,28,29]$, cellular morphology and development [30] and cellular aggregation [27]. In the latter process, three CBCRs cooperatively produce a light-color-sensitive input system for cyclic-di-GMP signaling that leads to cell aggregation [27].

\section{Far-red light photoacclimation (FaRLiP)}

FaRLiP is a newly discovered type of photoregulation, which controls the biosynthesis of specialized chlorophylls (Chls) and photosystems in some terrestrial cyanobacteria. This photoacclimation response allows these cyanobacteria to utilize FRL $(\lambda>700 \mathrm{~nm})$ for oxygenic photosynthesis and growth [19“;31-35]. FaRLiP was initially identified in Leptolyngbya sp. JSC1 and has subsequently been experimentally verified in several other species distributed across nearly the full taxonomic diversity of cyanobacteria $\left[19^{*}, 32,36\right]$. Under FRL-enriched conditions, which can be produced by filtering of light by Chl $a$, canopy effects or by scattering in soil, 
cyanobacteria that can perform FaRLiP (hereafter FaRLiP strains) synthesize Chl $d$, Chl $f$, longwavelength-absorbing forms of allophycocyanin (AP), and they use these unusual pigments to extensively remodel PSI, PSII, and PBS (Figures 3, 4) [19“,31-34“]. Comparative genomic analyses have shown that thirteen cyanobacteria have a conserved cluster of 20 genes, denoted as the FaRLiP cluster. This cluster encodes $\mathrm{Chl} f$ synthase (ChlF), transcriptional activators RfpA, $\mathrm{RfpB}$, and $\mathrm{RfpC}$, and paralogous copies of pigment-binding core subunits of PSI, PSII, and PBS $\left[19^{*}, 32-35,37^{\circ}\right]$. RfpA is a knotless phytochrome that covalently binds a single phycocyanobilin chromophore, which interconverts between its $\mathrm{P}_{\mathrm{r}}$ and $\mathrm{P}_{\mathrm{fr}}$ forms in response to RL and FRL, respectively (Figure 4A,B) [19"]. RfpA contains a histidine kinase, auto-phosphorylation domain. $\mathrm{RfpC}$, an essential CheY-like response regulator, is thought to act as a phosphate shuttle between RfpA and RfpB. RfpB, a response regulator that contains two CheY-like phosphorylation domains and a DNA-binding domain, is the transcriptional activator that ultimately controls the expression of the genes in the FaRLiP gene cluster in response to FRL (Figure 4) $\left[19^{*}, 35,37^{*}\right]$.

Chls $d$ and $f$ are FRL-absorbing Chls that have thus far only been identified in FaRLiP cyanobacteria $\left[19^{*}, 31,38^{*}-40\right]$. The $\mathrm{Q}_{\mathrm{y}}$ absorbance maxima in methanol for Chls $d$ and $f$ are 697 and $707 \mathrm{~nm}$, respectively [41]. Six FaRLiP strains have been experimentally verified to synthesize Chl $a$, Chl $d$ and $\mathrm{Chl} f$ when cells are grown in FRL (>700 nm); however, these strains only synthesize Chl $a$ when cells are grown in visible light wavelengths ( $\sim 400$ to $700 \mathrm{~nm}$ ) $\left[19^{*}, 32,34^{*}, 35,37^{\circ}\right]$. In addition to the thirteen FaRLiP strains identified by comparative genomics, a few other cyanobacterial strains also synthesize Chl $d$ and/or Chl $f\left[38^{*}, 39,42-49\right]$. Chl $d$ is the major pigment (>95\%) synthesized by Acaryochloris marina, but there is no evidence so far that its synthesis is regulated by FRL $[39,45,50,51]$. However, studies in other 
cyanobacterial species show that the synthesis of $\mathrm{Chl} d$ or $\mathrm{Chl} f$ occurs when cells are grown in FRL $\left[19^{*}, 32,34^{* *}, 35,37^{\circ}, 42,44,45,52\right]$. Although some candidates for enzymes involved in the synthesis of Chl $d$ have been proposed [53], the enzyme(s) responsible for its synthesis are currently unknown. Interestingly, small amounts of $\mathrm{Chl} d$ can be produced non-enzymatically from Chl $a$ in the presence of thiol compounds and oxygen [54-56] (M-Y Ho and DA Bryant, unpublished observations). This surprising observation suggests that a thiol ligase or protein cysteinyl lyase might be involved in Chl $d$ synthesis. The enzyme for the synthesis of Chl $f$ was also unknown until its recent identification as ChIF, formerly PsbA4, a protein encoded in all FaRLiP gene clusters [34"]. ChlF is an ancestral form of the PsbA core subunit of PSII, so-called super-rogue PsbA, which lacks the amino acid residues for binding a $\mathrm{Mn}_{4} \mathrm{Ca}_{1} \mathrm{O}_{5}$ cluster [57]. Instead of oxidizing water to produce dioxygen, ChlF apparently produces Chl $f$ (or chlorophyllide $f$ ) by photooxidation of $\mathrm{Chl} a$ (or chlorophyllide $a$ ) [34“]. RfpA, RfpB, and RfpC strongly activate the transcription of $c h l F / p s b A 4$, which explains the induction of $\mathrm{Chl} f$ synthesis when FaRLiP strains are grown in FRL $\left[19^{*}, 34^{*}, 35,37^{*}\right]$ (see Figure 4C). The $c h l F$ and $r f p A B C$ genes are universally present in the genomes of FaRLiP strains, and it seems likely that the genomes of other Chl $f$-producing cyanobacteria will have similar FaRLiP gene clusters.

Chl $d$ and Chl $f$ must associate with PSI and PSII to harvest FRL for photosynthesis. Paralogous copies of genes encoding pigment-binding core subunits of PSI and PSII facilitate Chl $d$ or Chl $f$ binding and the harvesting of FRL (see Figure 4C) [19“; M-Y Ho et al., unpublished results]. Similarly, the core subunits of the PBS are also replaced by FRL-absorbing AP subunits encoded in the FaRLiP cluster $\left[19^{*}, 33,36,58,59\right]$. Notably, AP subunits synthesized in white light (WL) and AP subunits encoded in the FaRLiP cluster both bind phycocyanobilin, although ApcE2 produced in FRL does so non-covalently, which leads to a red-shift of the 
absorption because of the presence of one additional conjugated double bond in the chromophore $\left[19^{*}, 36,58\right]$. To harvest FRL efficiently, PBS in three different cyanobacterial species are either remodeled by forming complexes with bicylindrical cores and peripheral rods, by solely forming bicylindrical cores, or by retaining a combination of bicylindrical cores and RL-absorbing PBS $\left[19^{*}, 33,36\right]$. Like $\mathrm{Chl} f$ synthesis, the expression of genes required for remodeling of PSI, PSII, and PBS is regulated by $\mathrm{RfpA}, \mathrm{RfpB}$, and $\mathrm{RfpC}$ [35,37']. In summary, FRL activates the syntheses of $\mathrm{Chl} d$ and $\mathrm{Chl} f$ in FaRLiP strains and additionally leads to the structural remodeling of PSI, PSII, and PBS. These changes optimize the photosynthetic apparatus for harvesting FRL for oxygenic photosynthesis and growth (Figures 3, 4) [19*0].

\section{Complementary Chromatic Acclimation (CCA)}

CCA refers to the light wavelength (color)-dependent alteration of phycobiliproteins that occur in the peripheral rods of PBS, the light-harvesting antenna complexes of cyanobacteria (Figure 3). In these cyanobacteria light wavelength controls the expression of the genes encoding phycoerythrin (PE) and phycocyanin (PC) that make up the peripheral rods of PBSs, as well as assembly-related proteins (linker proteins) and enzymes for the synthesis of the bilin chromophores that are bound to these phycobiliproteins. These changes improve cellular light harvesting by increasing the overlap between the incident light and the absorption cross-section of PBS [60]. As noted above, the discovery of numerous CBCRs has revealed that cyanobacteria have the potential to regulate gene expression in response to light spanning the range from the NUV to the NIR (see Introduction). In CCA this capability is applied to the production of bilins and phycobiliproteins. Because of the powerful selective advantage gained by improvements in light harvesting for organisms that are strictly phototrophic, it is not surprising that diverse members of the phytochrome/CBCR superfamily have evolved to regulate this process. 
The various CCA strategies employed by cyanobacteria are broadly grouped into four categories, denoted Types I-IV CCA [60,61“]. In Type I organisms, the expression of PC and PE is not regulated by light wavelength. In Type II organisms, PE synthesis occurs in GL or WL but not in RL, and PC synthesis is not regulated. In Type III organisms, the synthesis of both PE and PC are regulated in response to GL and RL, respectively. In Type IV organisms, the chromophore content of phycoerythrin-II (PE-II) is modified to harvest BL or GL better. Recent studies of Types III and IV CCA have revealed new complexity in their photoregulation.

Cyanobacteria capable of Type III CCA synthesize more PE in GL compared to RL, and more PC in RL compared to GL (Figure 3) [60]. When organized in the peripheral rods of PBSs, these phycobiliproteins specifically increase light harvesting of the complementary light colors, GL and OL/RL, respectively. The master-regulator of Type III CCA in F. diplosiphon is a GL/RL-responsive CBCR, RcaE, which modulates expression of the genes encoding PE (cpeBA) and PC-2 ( $c p c B 2 A 2)$ and their associated linker polypeptides [62]. A similar photoreceptor, CcaS, controls PE synthesis in GL in Type II CCA in $N$. punctiforme $\left[26^{\circ}, 63\right]$. RcaE signaling in GL increases the expression of another CBCR, IflA [64]. IflA contains two chromophorylated GAF domains that together allow this photoreceptor to integrate information concerning four colors of light: BL, GL, RL and FRL. Deletion of iflA reduces the growth rates of low-density cultures of F. diplosiphon, suggesting that IflA senses the RL:FRL ratio in the environment and accelerates growth at low cell density. These observations suggest a new role for RcaE beyond its role in regulating Type III CCA and further suggest that a complex network of CBCRs regulates CCA [64].

The concept of a regulatory $\mathrm{CBCR}$ network for CCA was further expanded with the discovery of the TL/YL-responsive protein, DpxA, in F. diplosiphon [65 ]. DpxA represses PE 
accumulation in YL but not in TL, adding finer control over the RcaE-mediated repression of PE in GL and preventing the deterioration of Type III CCA at near-green wavelengths to which RcaE does not respond optimally [65"]. The discovery of IflA and DpxA reveal a higher level of complexity for the regulation of Type III CCA by light. Similar to phototaxis [11,29] and cell aggregation [27], multiple CBCR photoreceptors are employed to achieve optimal control.

Type IV CCA remains the least characterized CCA mechanism. Marine Synechococcus species that can perform Type IV CCA alter the bilin chromophore(s) that are attached to PE-II [61"]. When cells are grown in BL, PE-II is predominantly chromophorylated with phycourobilin (PUB), while phycoerythrobilin (PEB) is the predominant chromophore attached to PE-II when cells are grown in GL $\left[60,61^{*}\right]$. The changes in chromophore content optimize the light-harvesting capacity of PE-II for each condition. The transcriptional regulators responsible for tuning the PUB:PEB ratio in response to BL and GL were recently identified as FciA and FciB. Inactivation of either $f c i A$ or $f c i B$ caused cells to adopt permanently a GL- or BLphenotype, respectively [61“]. FciA activates the expression of three genes in BL, including the gene encoding the lyase/isomerase, MpeZ, which attaches PUB to the alpha subunit of PE-II. FciB represses the transcription of these three genes in GL. This co-regulated genomic island was likely acquired by various marine Synechococcus spp. through horizontal gene transfer. Surprisingly, none of the genes in this operon encodes a protein with a known photosensing domain, so the connection between light color and gene expression in Type IV CCA is still a mystery, as are the functions of the other two co-regulated genes, $f c i C$ and unk10 [61“].

\section{Other light-regulated processes affecting the photosynthetic}

\section{apparatus in cyanobacteria}


LoLiP describes the ability of some Synechococcus strains to alter their photosynthetic apparatus in order to absorb far-red light after growth at low irradiance $\left[31,66^{\circ}\right]$ (Figure 1). Lowlight (LL)-adapted and high-light (HL)-adapted ecotypes of Synechococcus species from hot spring microbial mats exhibited different responses to HL and LL [66 ${ }^{\circ}$. Comparative genomics of HL- and LL-adapted strains revealed an operon containing four genes that is exclusively found in the genomes of the LL-adapted ecotypes. The genes are predicted to encode a putative $\mathrm{CBCR}$, the alpha and beta subunits of an $\mathrm{AP}$ variant ( $a p c D 4$ and $a p c B 3$, respectively) and $i s i X$, a homolog of the iron-stress-inducible, Chl $a$-binding IsiA protein [67"]. The AP genes in this operon produce a FRL-absorbing AP variant and contribute to the FRL absorbance observed in LL-adapted Synechococcus sp. cells after growth at low irradiances, but how these proteins are assembled into the photosynthetic apparatus has not yet been established (NT Soulier and DA Bryant, unpublished results).

The cyanobacterial circadian clock rhythmically controls gene expression patterns governing cellular physiology, allowing cells to adapt to predictable environmental fluctuations occurring on a daily basis - most notably changes in temperature and light—in a manner that is independent of cell cycle phase [for a recent review, see 68]. This clock is controlled by phosphorylation of oscillator proteins, encoded by the kaiABC genes, which receive signals indicating a change in external environmental conditions via regulatory information transmission from light- and redox-sensitive proteins such as CikA and LdpA [68]. The Kai complex/circadian clock appears to affect transcription globally by rhythmically compacting the chromosomal DNA in cells [68]. In one half of the diel cycle, the compacted chromosome is transcriptionally inaccessible, while in the following half this compaction is reversed, allowing the transcriptional machinery to function. CikA is a CBCR in some cyanobacteria and has a GAF 
domain that binds phycocyanobilin by two thioether linkages and absorbs maximally at $\sim 415 \mathrm{~nm}$ $\left[9,11,15,21^{\circ}, 24\right]$. Thus, light can directly play a role in regulating circadian processes in some cases. Recently, reconstruction of the circadian oscillator in a non-circadian model organism, Escherichia coli, demonstrated oscillations in KaiC phosphorylation and in expression of a synthetic transcriptional reporter. This achievement establishes the minimally sufficient set of proteins required for circadian control of transcriptional output, which opens up the possibility for introduction of circadian control into heterologous systems [69"].

Other processes, such as the overall cellular content of components of the photosynthetic apparatus and adjustments to the PSI:PSII ratio, also respond to light color and intensity [70 -73$]$. Although it is known that sensor kinases for stresses affect these adjustments, it is not known whether specific photoreceptors are involved in the long-term adaptation/acclimation of the photosynthetic apparatus. Similarly, short-term acclimation of the photosynthetic apparatus (state transitions) involves rapid changes in the specific associations of PSI, PSII, and PBS [74,75" $]$. Similarly, short-term acclimation of the photosynthetic apparatus, i.e. state transitions, induced by changes in light color/wavelength, involve relative movements of PBS and/or photosystems that modify the contacts between PBS and the two photosystems as well as between PSII and PSI [76,77]. Although the redox state of the plastoquinoine pool is thought to regulate these processes, a $\mathrm{CBCR}$ photoreceptor similar to $\mathrm{CcaS}$ is involved in controlling the synthesis of a membrane-anchored PC-CpcG2 rod complex that associates with PSI [78*].

\section{Concluding Remarks}

Genome sequencing and bioinformatic analyses have led to a rapid expansion of our knowledge of photoreceptors in cyanobacteria. Coupled with insightful functional and structural studies, 
rapid progress is now being made in elucidating their roles in diverse photoreceptors in various physiological and metabolic processes. It is important that cyanobacteria that have not been used traditionally as laboratory model organisms among cyanobacteria receive adequate study. Filamentous cyanobacteria are physiologically and developmentally complex and tend to have much larger genomes than unicellular cyanobacteria, and correspondingly they have greater numbers of uncharacterized photoreceptor. It is possible to apply genetic methods, especially conjugation, to the study of some of these organisms $\left[35,37^{\circ}, 60\right]$. Another approach that can now be used, but that has been underutilized, is to study the ecophysiological role of light in natural cyanobacterial communities (e.g., hot spring mat or hypersaline mat communities). It should be possible to manipulate the incident light in ways that can reveal the roles of photoreceptors in diverse processes in situ, and thereby gain insights into the light niches and selective pressures that led to the evolution of various photoadaptive and photoacclimation process as described above. The FaRLiP and LoLiP processes described here were discovered through a combination of in situ and ex situ studies. An especially powerful approach is to combine field studies using omic methods with ex situ studies of truly representative organisms isolated from the same microbial community. As our collaborator Dr. David M. Ward at Montana State University, often remarks, it is always a good idea to "let Nature be your guide."

\section{Acknowledgements}

Work on light-harvesting in cyanobacteria, especially far-red light photoacclimation has been supported by National Science Foundation grants MCB-1021725 and MCB-1613022 to D.A.B This research was also conducted under the auspices of the Photosynthetic Antenna Research Center (PARC), an Energy Frontier Research Center funded by the DOE, Office of Science, Office of Basic Energy Sciences under Award Number DE-SC 0001035. M.-Y.H., G.S, N.T.S., and D.A.B. were partly supported by PARC and partly supported by N.S.F. D.P.C. is supported by a Marie Skłodowska-Curie Global Fellowship (660652) from the European Commission. 


\section{References and recommended reading:}

Papers of particular interest, published within the period of review, have been highlighted as:

- of special interest

.. of outstanding interest

1. Grotjohann I, Fromme P: Structure of cyanobacterial photosystem I. Photosynth Res 2005, 85:51-72.

2. Nelson N, Junge $\mathrm{W}$ : Structure and energy transfer in photosystems of oxygenic photosynthesis. Annu Rev Biochem 2015, 84:659-683.

3. Shen J-R: The structure of Photosystem II and the mechanism of water oxidation in photosynthesis. Annu Rev Plant Biol 2015, 66:559-568.

4. Sidler W: Phycobilisome and phycobiliprotein structures. In: Advances in Photosynthesis and Respiration, Vol 1, The Molecular Biology of Cyanobacteria. Edited by Bryant DA. Springer; 1994:139-216.

5. Cramer WA, Hasan SS, Yamashita E: The $\mathbf{Q}$ cycle of cytochrome bc complexes: a structure perspective. Biochim Biophys Acta 2011, 1807:788802.

6. Goss T, Hanke G: The end of the line: can ferredoxin and ferredoxinNADP $(H)$ oxidoreductase determine the fate of photosynthetic electrons? Curr Protein Pept Sci 2014, 15:385-393.

7. Junge W, Nelson N: ATP synthase. Annu Rev Biochem 2015, 84:631-657.

8. Yerrapragada S, Shukla A, Hallsworth-Pepin K, Choi K, Wollam A, Clifton S, Qin X, Muzny D, Raghuraman S, Ashki H, Uzman A, Highlander SK, Fryszczyn BG, Fox GE, Tirumalai MR, Liu Y, Kim S, Kehoe DM, Weinstock GM: Extreme sensory complexity encoded in the 10-megabase draft genome sequence of the chromatically acclimating cyanobacterium Tolypothrix sp. PCC 7601. Genome Announc 2015, 3:e00355-15. doi:10.1128/genomeA.00355-15

9. Rockwell NC, Martin SS, Gan F, Bryant DA, Lagarias JC: NpR3784 is the prototype for a distinctive group of red/green cyanobacteriochromes using 
alternative Phe residues for photoproduct tuning. Photochem Photobiol Sci 2015, 14:258-269.

10. Cho SM, Jeoung, SC, Song J-Y, Kupriyanova EV, Pronina NA, Lee B-W, Jo SW, Park B-S, Choi S-B, Song J-J, and Park Y-I: Genomic survey and biochemical analysis of recombinant candidate cyanobacteriochromes reveals enrichment for near-UV/Violet sensors in the halotolerant and alkaliphilic cyanobacterium Microcoleus IPPAS B353. J Biol Chem 2015, 290:28502-28514.

11. Ikeuchi $\mathrm{M}$, Ishizuka $\mathrm{T}$ : Cyanobacteriochromes: a new superfamily of tetrapyrrole-binding photoreceptors in cyanobacteria. Photochem Photobiol Sci 2008, 7:1159-1167.

-12. Rockwell NC, Martin SS, Feoktistova K, Lagarias JC: Diverse two-cysteine photocycles in phytochromes and cyanobacteriochromes. Proc Natl Acad Sci USA 2011, 108:11854-11859.

This study documents the remarkable flexibility of dual-Cys GAF domains of phytochromes and cyanobacteriochromes for sensing light of different wavelengths.

13. Rockwell NC, Martin SS, Lagarias JC: Identification of cyanobacteriochromes detecting far-red light. Biochemistry 2016, 55:3907-3919.

14. Quest B, Hübschmann T, Sharda S, Tandeau de Marsca N, Gärtner W: Homologous expression of a bacterial phytochrome. The cyanobacterium Fremyella diplosiphon incorporates biliverdin as a genuine, functional chromophore. FEBS J 2007, 274:2088-2098.

15. Rockwell NC, Martin SS, Gulevich AG, Lagarias JC: Phycoviolobilin formation and spectral tuning in the DXCF cyanobacteriochrome subfamily. Biochemistry 2012, 51:1449-1463.

16. Burgie ES, Vierstra RD: Phytochromes: an atomic perspective on photoactivation and signaling. Plant Cell 2014, 26:4568-4583.

17. Rockwell NC, Lagarias JC: A brief history of phytochromes. ChemPhysChem 2010, 11:1172-1180. 
18. Rockwell NC, Martin SS, Lagarias JC: Identification of cyanobacteriochromes detecting far-red light. Biochemistry 2016, 55:3907-3919.

• 19. Gan F, Zhang S, Rockwell NC, Martin SS, Lagarias JC, Bryant DA: Extensive remodeling of a cyanobacterial photosynthetic apparatus in far-red light. Science 2014, 345:1312-1317.

The full complexity of far-red light photoacclimation (FaRLiP) of was first described in this article.

20. Rockwell NC, Martin SS, Lagarias JC: Red/green cyanobacteriochromes: sensor of color and power. Biochemistry 2012, 51:9667-9677.

-21. Fushimi K, Rockwell NC, Enomoto G, Win N-N, Martin SS, Gan F, Bryant DA, Ikeuchi M, Lagarias JC, Narikawa R: Cyanobacteriochrome photoreceptors lacking the canonical Cys residue. Biochemistry 2016, doi:10.1021/acs.biochem.6b00940.

This study shows that a novel, unknown chromophore binds to CBCR photoreceptors that lack the canonical cysteine residue for attachment at $\mathrm{C}-3^{1}$ but still have the second cysteine residue that can bind to the $\mathrm{C}-10$ position of the chromophore.

22. Rockwell NC, Njuguna SL, Roberts L, Castillo E, Parson VL, Dwojak S, Lagarias JC, Spiller SC: A second conserved GAF domain cysteine is required for the blue/green reversibility of cyanobacteriochrome Tlr0924 from Thermosynechococcus elongatus. Biochemistry 2008, 47:7304-7316.

23. Rockwell NC, Martin SS, Lagarias JC: Mechanistic insight into the photosensory versatility of DXCF cyanobacteriochromes. Biochemistry 2012, 51:3576-3585.

24. Rockwell NC, Martin SS, Lagarias JC: Identification of DXCF cyanobacteriochrome lineages with predictable photocycles. Photochem Photobiol Sci 2015, 14:929-941.

••25. Burgie ES, Walker JM, Phillips GNJ, Vierstra RD: A photo-labile thioether linkage to phycoviolobilin provides the foundation for blue/green photocycles in DXCF-cyanobacteriochromes. Structure 2013, 21:88-97. 
The structure of the GAF domain of PixJ from Thermosynechococcus elongatus BP-1 is presented in this study. This study verified the existence of the second thioether linkage to the chromophore at position $\mathrm{C}-10$.

-26. Hirose Y, Rockwell NC, Nishiyama K, Narikawa R, Ukaji Y, Inomata K, Ikeuchi M: Green/red cyanobacteriochromes regulate complementary chromatic acclimation via a protochromic photocycle. Proc Natl Acad Sci USA 2013, 110:4974-4979.

This study showed that isomerization of the chromophore alone was not responsible for the altered absorption of RcaE, but that protonation of the bilin after isomerization played the key role in the "protochromic" photocycle of this photoreceptor.

27. Enomoto G, Narikawa R, Ikeuchi M: Three cyanobacteriochromes work together to form a light color-sensitive input system for c-di-GMP signaling of cell aggregation. Proc Natl Acad Sci USA 2015, 112:8082-8087.

28. Campbell EL, Hagen KD, Chen R, Risser DD, Ferreira DP, Meeks JC: Genetic analysis reveals the identity of the photoreceptor for phototaxis in hormogonium filaments of Nostoc punctiforme. J Bacteriol 2015, 197:782791.

29. Song JY, Cho HS, Cho JI, Jeon JS, Lagarias JC, Park YI: Near-UV cyanobacteriochrome signaling system elicits negative phototaxis in the cyanobacterium Synechocystis sp. PCC 6803. Proc Natl Acad Sci USA 2011, 108:10780-10785.

30. Montgomery BL: Mechanisms and fitness implications of photomorphogenesis during chromatic acclimation in cyanobacteria. $J$ Exp Bot 2016, 67:4079-4090.

31. Gan F, Bryant DA: Adaptive and acclimative responses of cyanobacteria to far-red light. Environ Microbiol 2015, 17:3450-3465.

32. Gan F, Shen G, Bryant D: Occurrence of far-red light photoacclimation (FaRLiP) in diverse cyanobacteria. Life 2015, 5:4-24.

33. Ho M-Y, Gan F, Shen G, Bryant DA: Far-red light photoacclimation (FaRLiP) in Synechococcus sp. PCC 7335. II. Characterization of phycobiliproteins 
produced during acclimation to far-red light. Photosynth Res 2016, doi: 10.1007/s11120-016-0303-5.

•.34. Ho M-Y, Shen G, Canniffe DP, Zhao C, Bryant DA: Light-dependent chlorophyll $f$ synthase is a highly divergent paralog of PsbA of Photosystem II. Science 2016, 353:aaf9178. doi: 10.1126/science.aaf9178

The authors identified $\mathrm{Chl} f$ synthase (ChIF), which catalyzes $\mathrm{Chl} f$ formation in a lightdependent manner. The chIF gene, formerly annotated as $p s b A 4$, was shown to be present in FaRLiP gene cluster. ChIF is a distant and probably ancestral paralog of the PsbA core subunit of PSII.

35. Ho M-Y, Gan F, Shen G, Zhao C, Bryant DA: Far-red light photoacclimation (FaRLiP) in Synechococcus sp. PCC 7335: I. Regulation of FaRLiP gene expression. Photosynth Res 2016, doi: 10.1007/s11120-016-0309-z.

36. Li Y, Lin Y, Garvey CJ, Birch D, Corkery RW, Loughlin PC, Scheer H, Willows $R D$, Chen $M$ : Characterization of red-shifted phycobilisomes isolated from the chlorophyll f-containing cyanobacterium Halomicronema hongdechloris. Biochim Biophys Acta 2016, 1857:107-114.

-37. Zhao C, Gan F, Shen G, Bryant DA: RfpA, RfpB, and RfpC are the master control elements of far-red light photoacclimation (FaRLiP). Front Microbiol 2015, 6:1303.

This study established the functions of $\operatorname{RfpA}$, RfpB, and $\mathrm{RfpC}$ in transcriptional regulation of the FaRLiP gene cluster in two cyanobacteria.

••38. Chen M, Schliep M, Willows RD, Cai Z-L, Neilan BA, Scheer H: A red-shifted chlorophyll. Science 2010, 329:1318-1319.

This important study describes the discovery of chlorophyll $f$ in cyanobacteria.

39. Chen M: Chlorophyll modifications and their spectral extension in oxygenic photosynthesis. Annu Rev Biochem 2014, 83:317-340.

40. Allakhverdiev SI, Kreslavski VD, Zharmukhamedov SK, Voloshin RA, Korol'kova DV, Tomo T, Shen JR: Chlorophylls $d$ and $f$ and their role in primary 
photosynthetic processes of cyanobacteria. Biochemistry (Moscow) 2016, 81:201-212.

41. Li Y, Scales N, Blankenship RE, Willows RD, Chen M: Extinction coefficient for red-shifted chlorophylls: Chlorophyll $\boldsymbol{d}$ and chlorophyll $\boldsymbol{f}$. Biochim Biophys Acta 2012, 1817:1292-1298.

42. Chen $\mathrm{M}$, Li $\mathrm{Y}$, Birch $\mathrm{D}$, Willows $\mathrm{RD}$ : A cyanobacterium that contains chlorophyll $f$ - a red-absorbing photopigment. FEBS Lett 2012, 586:3249-3254.

43. Akutsu S, Fujinuma D, Furukawa H, Watanabe T, Ohnishi-Kameyama M, Ono H, Ohkubo S, Miyashita H, Kobayashi M: Pigment analysis of a chlorophyll $f$ containing cyanobacterium strain KC1 isolated from Lake Biwa. Photomed Photobiol 2011, 33:35-40.

44. Airs RL, Temperton B, Sambles C, Farnham G, Skill SC, Llewellyn CA: Chlorophyll $f$ and chlorophyll $d$ are produced in the cyanobacterium Chlorogloeopsis fritschii when cultured under natural light and near-infrared radiation. FEBS Lett 2014, 588:3770-3777.

45. Miyashita $H$, Ohkubo $S$, Komatsu $H$, Sorimachi $Y$, Fukayama D, Fujinuma D, Akutsu S, Kobayashi M: Discovery of chlorophyll d in Acaryochloris marina and chlorophyll $f$ in a unicellular cyanobacterium, strain KC1, Isolated from Lake Biwa. J Phys Chem Biophys 2014, 4:149. doi:10.4172/2161-0398.1000149

46. Trampe E, Kühl M: Chlorophyll $f$ distribution and dynamics in cyanobacterial beachrock biofilms. J Phycol 2016, 52:990-996.

47. Behrendt L, Brejnrod A, Schliep M, Sørensen SJ, Larkum AWD, Kühl M: Chlorophyll $f$-driven photosynthesis in a cavernous cyanobacterium. ISME J 2015, 9:2108-2111.

48. Miyashita H, Ikemoto H, Kurano N, Adachi K, Chihara M, Miyachi S: Chlorophyll d as a major pigment. Nature 1996, 383:402.

49. Murakami A, Miyashita $\mathrm{H}$, Iseki M, Adachi K, Mimuro M: Chlorophyll d in an epiphytic cyanobacterium of red algae. Science 2004, 303:1633.

50. Miyashita H, Adachi K, Kurano N, Ikemot H, Chihara M, Miyach S: Pigment composition of a novel oxygenic photosynthetic prokaryote containing chlorophyll $\boldsymbol{d}$ as the major chlorophyll. Plant Cell Physiol 1997, 38:274-281. 
51. Li Y, Chen M: Novel chlorophylls and new directions in photosynthesis research. Funct Plant Biol 2015, 42:493-501.

52. Itoh S, Ohno T, Noji T, Yamakawa H, Komatsu H, Wada K, Kobayashi M, Miyashita $\mathrm{H}$ : Harvesting far-red light by chlorophyll $\boldsymbol{f}$ in photosystems I and II of unicellular cyanobacterium strain KC1. Plant Cell Physiol 2015, 56:20242034.

53. Yoneda A, Wittmann BJ, King JD, Blankenship RE, Dantas G: Transcriptomic analysis illuminates genes involved in chlorophyll synthesis after nitrogen starvation in Acaryochloris sp. CCMEE 5410. Photosynth Res 2016, 129:171182.

54. Fukusumi T, Takei N, Tateno Y, Aoki T, Ando A, Kozakai K, Shima H, Mizoguchi T, Ito $\mathrm{S}$, Ikeda $\mathrm{T}$, et al.: Ene-thiol reaction of C3-vinylated chlorophyll derivatives in the presence of oxygen: synthesis of C3-formyl-chlorins under mild conditions. J Porphyrins Phthalocyanines 2013, 17:1188-1195.

55. Fukusumi T, Matsuda K, Mizoguchi T, Miyatake T, Ito S, Ikeda T, Tamiaki H, Oba T: Non-enzymatic conversion of chlorophyll-a into chlorophyll-d in vitro: A model oxidation pathway for chlorophyll-d biosynthesis. FEBS Lett 2012, 586:2338-2341.

56. Loughlin PC, Willows RD, Chen M: In vitro conversion of vinyl to formyl groups in naturally occurring chlorophylls. Sci Rep 2014, 4:6069. doi:10.1038/srep06069

57. Murray JW: Sequence variation at the oxygen-evolving centre of photosystem II: a new class of 'rogue' cyanobacterial D1 proteins. Photosynth Res 110:177-184.

58. Miao D, Ding W-L, Zhao B-Q, Lu L, Xu Q-Z, Scheer H, Zhao K-H: Adapting photosynthesis to the near-infrared: non-covalent binding of phycocyanobilin provides an extreme spectral red-shift to phycobilisome core-membrane linker from Synechococcus sp. PCC7335. Biochim Biophys Acta 2016, 1857:688-694.

59. Xu Q-Z, Han J-X, Tang Q-Y, Ding W-L, Miao D, Zhou M, Scheer H, Zhao K-H: Far-red light photoacclimation: Chromophorylation of FR induced $\alpha$ - and $\beta$ - 
subunits of allophycocyanin from Chroococcidiopsis thermalis sp. PCC7203. Biochim Biophys Acta 2016, 1857:1607-1616.

60. Gutu A., Kehoe DM: Emerging perspectives on the mechanisms, regulation, and distribution of light color acclimation in cyanobacteria. Mol Plant 2012, 5:1-13.

••61. Sanfilippo JE, Nguyen AA, Karty JA, Shukla A, Schluchter WM, Garczarek L, Partensky F, Kehoe DM: Self-regulating genomic island encoding tandem regulators confers chromatic acclimation to marine Synechococcus. Proc Natl Acad Sci USA 2016, 113:6077-6082.

This study establishes that a small genomic island encoding five genes is responsible for the regulation and induction of Type IV CCA in marine Synechococcus spp.

62. Terauchi K, Montgomery BL, Grossman AR, Lagarias JC, Kehoe DM: RcaE is a complementary chromatic adaptation photoreceptor required for green and red light responsiveness. Mol Microbiol 2004, 51:567-577.

63. Hirose $\mathrm{Y}$, Narikawa R, Katayama M, Ikeuchi M: Cyanobacteriochrome CcaS regulates phycoerythrin accumulation in Nostoc punctiforme, a group II chromatic adapter. Proc Natl Acad Sci USA 2010, 107:8854-8859.

64. Bussell AN, Kehoe DM: Control of a four-color sensing photoreceptor by a two-color sensing photoreceptor reveals complex light regulation in cyanobacteria. Proc Natl Acad Sci USA 2013, 110:12834-2839.

-65. Wiltbank LB, Kehoe DM: Two cyanobacterial photoreceptors regulate photosynthetic light harvesting by sensing teal, green, yellow, and red light. mBio 2016, 7:e02130-15. doi:10.1128/mBio.02130-15

This study expands our understanding how Type III CCA is regulated by red and green light by showing that a second photoreceptor fine-tunes the acclimative response to teal and yellow light.

•66. Nowack S, Olsen MT, Schaible GA, Becraft ED, Shen G, Klapper I, Bryant, DA Ward DM: The molecular dimension of microbial species: 2. Synechococcus strains representative of putative ecotypes inhabiting 
different depths in the Mushroom Spring microbial mat exhibit different adaptive and acclimative responses to light. Front Microbiol 2015, 6:626. doi: 10.3389/fmicb.2015.00626

This study compares the HL tolerance and capacity for growth at LL for both HLadapted and LL-adapted putative ecotype strains of Synechococcus sp. recovered from the mats of Mushroom Spring in Yellowstone National Park, WY.

•67. Olsen MT, Nowack S, Wood JM, Becraft ED, Labutti K, Lipzen A, Martin J, Schackwitz WS, Rusch DB, Cohan FM, Bryant DA, and Ward DM. The molecular dimension of microbial species: 3. Comparative genomics of Synechococcus strains with different light responses and in situ diel transcription patterns of associated putative ecotypes in the Mushroom Spring microbial mat. Front Microbiol 2015, 6:604. doi: 10.3389/fmicb.2015.00604

This study describes the genetic differences between HL-adapted and LL-adapted putative ecotype strains of Synechococcus sp. The comparative genomics led to the discovery of a four-gene operon, encoding a gene for putative CBCR and apcD4apcB3-isiX, that occurs in the LL-adapted ecotypes but that is missing from the HLadapted ecotypes.

68. Cohen SE, Golden SS: Circadian rhythms in cyanobacteria. Microbiol Mol Biol Rev 2015, 79:373-85.

••69. Chen AH, Lubkowicz D, Yeong V, Chang RL, Silver PA: Transplantability of a circadian clock to a noncircadian organism. Sci Adv 2015, 1:e1500358. doi: 10.1126/sciadv.1500358

Reconstruction of the oscillator in Escherichia coli shows circadian rhythm in KaiC phosphorylation and for a synthetic transcriptional reporter. The minimal set of proteins for circadian transcriptional output is defined.

-70. Muramatsu M, Hihara $\mathrm{Y}$ : Acclimation to high-light conditions in cyanobacteria: from gene expression to physiological responses. J Plant Res 2012, 125:11-39. 
This review provides an explanation for the acclimation response to high light based upon gene expression and photosystem biogenesis in cyanobacteria, which mostly serve to balance light harvesting and optimize electron transport.

71. Kopečná J, Komenda J, Bučinská L, Sobotka R: Long-term acclimation of the cyanobacterium Synechocystis sp. PCC 6803 to high light is accompanied by an enhanced production of chlorophyll that is preferentially channeled to trimeric photosystem I. Plant Physiol 2012, 160:2239-2250.

72. Seino $Y$, Takahashi $T$, Hihara $Y$ : The response regulator RpaB binds to the upstream element of photosystem I genes to work for positive regulation under low-light conditions in Synechocystis sp. Strain PCC 6803. J Bacteriol 2009, 191:1581-1586.

73. Sobotka R: Making proteins green; biosynthesis of chlorophyll-binding proteins in cyanobacteria. Photosynth Res 2014, 119:223-232.

74. Dong C, Tang A, Zhao J, Mullineaux CW, Shen G, Bryant DA: ApcD is necessary for efficient energy transfer from phycobilisomes to photosystem $I$ and helps to prevent photoinhibition in the cyanobacterium Synechococcus sp. PCC 7002. Biochim Biophys Acta 2009, 1787:1122-1128.

••75. Liu H, Zhang H, Niedzwiedzki DM, Prado M, He G, Gross ML, Blankenship RE: Phycobilisomes supply excitations to both photosystems in a megacomplex in cyanobacteria. Science 2013, 342:1104-1107.

This mass spectrometric study directly confirmed the interactions of ApcE with PS II and $A p c D$ with PSI, which verified predictions based upon gene inactivation studies.

76. Mullineaux CW: Phycobilisome-reaction centre interaction in cyanobacteria. Photosynth Res 2008, 95:175-182.

77. Mullineaux CW: Electron transport and light-harvesting switches in cyanobacteria. Front Plant Sci 2014, 5:7 doi: 10.3389/fpls.2014.00007

-78. Watanabe M, Semchonok DA, Webber-Birungi MT, Ehira S, Kondo K, Narikawa $\mathrm{R}$, Ohmori M, Boekema EJ, Ikeuchi M: Attachment of phycobilisomes in an 
antenna-photosystem I supercomplex of cyanobacteria. Proc Natl Acad Sci USA 2014, 111:2512-2517.

This study showed that a specialized rod-core linker, $\mathrm{CpcG} 3 / \mathrm{CpcL}$, assembled phycocyanin rods that associate with PSI complexes to form a phycobiliprotein/PSI supercomplex. 


\section{Figure Legends}

Figure 1. Network of light regulation and its impact on photosynthetic components in cyanobacteria. Far-red light photoacclimation (FaRLiP), complementary chromatic acclimation (CCA), low-light photoacclimation (LoLiP), circadian rhythm, PSI and PSII content and ratio, and state transitions affect the biosynthesis, biogenesis and functionality of different photosynthetic components and complexes. Solid lines represent verified connections. Dashedlines indicate uncertain relationships.

Figure 2. Chromophore structure of the GAF domain of PixJ1 from Thermosynechococcus elongatus BP-1, a BL/GL photoreceptor [25"]. A. Phycoviolobilin structure with thioether linkages at carbons C- $3^{1}$ and C-10. B. Details of the binding pocket for phycoviolobilin showing the thioether linkages provided by cysteines 522 and 494 of PixJ. C. Structure of the phycoviolobilin binding pocket of PixJ1 (for additional details, see [25*]. The structures in panels $\mathbf{B}$ and $\mathbf{C}$ were modified from RSCB PDB accession number 4FOF (for additional details, see $\left.\left[25^{\circ}\right]\right)$. D. Appearance of the GAF domain of PixJ1 in solution in its $\mathrm{Pb}$ and Pg forms (top), and absorbance spectra of the two forms of the protein (below). Panel D is adapted from [11].

Figure 3. Acclimation of Synechococcus sp. PCC 7335 to different light conditions. Synechococcus sp. PCC 7335 is a cyanobacterium that performs both complementary chromatic acclimation (CCA) and far-red light photoacclimation (FaRLiP). The absorbance spectra were taken for Synechococcus sp. PCC 7335 cultured in white light (WL), red light (RL), green light (GL), and far-red light (FRL) and were normalized at $750 \mathrm{~nm}$. Production of phycoerythrin (PE) is induced in WL and GL, while the synthesis of PC is reduced in GL. In RL, more phycocyanin (PC) is produced, and synthesis of $\mathrm{PE}$ is downregulated. FRL induces syntheses of $\mathrm{Chl} d, \mathrm{Chl} f$, and FRL-absorbing allophycocyanin subunits (AP). Please see references [33-35] and the text for additional details.

Figure 4. FRL $(\lambda>700 \mathrm{~nm})$ regulates FaRLiP through RfpABC-dependent and independent pathways. (A). Appearance of recombinant $\mathrm{RfpA}$ in its $\mathrm{P}_{\mathrm{r}}$ and $\mathrm{P}_{\mathrm{fr}}$ forms. (B). Absorption spectra of recombinant GAF domain of RfpA after $700 \mathrm{~nm}$ or $645 \mathrm{~nm}$ illumination (nearly identical results were obtained with the full-length protein). (C). Scheme of FRLregulated pigment biosynthesis and remodeling of the photosynthetic apparatus. FRL 
photoconverts $\mathrm{RfpA}$ from $\mathrm{P}_{\text {fr }}$ form to $\mathrm{P}_{\mathrm{r}}$ form, activating its histidine auto-kinase, which phosphorylates RfpC. Acting as a phosphate shuttle, RfpC phosphorylates the response regulator/transcriptional activator, $\mathrm{RfpB}$. Genes involved in chlorophyll (Chl) biosynthesis and remodeling of PSI, PSII, and PBS are induced in RfpABC-dependent (black arrows) and RfpABC-independent (white arrows) pathways. Note that although this figure shows the active form of RfpB is phosphorylated, the active forms of all of the proteins could also be the dephosphorylated state. Panel B is modified from [19“"]. Panel C is modified from [37"] with updated results from [34"*]. 
Figure 1

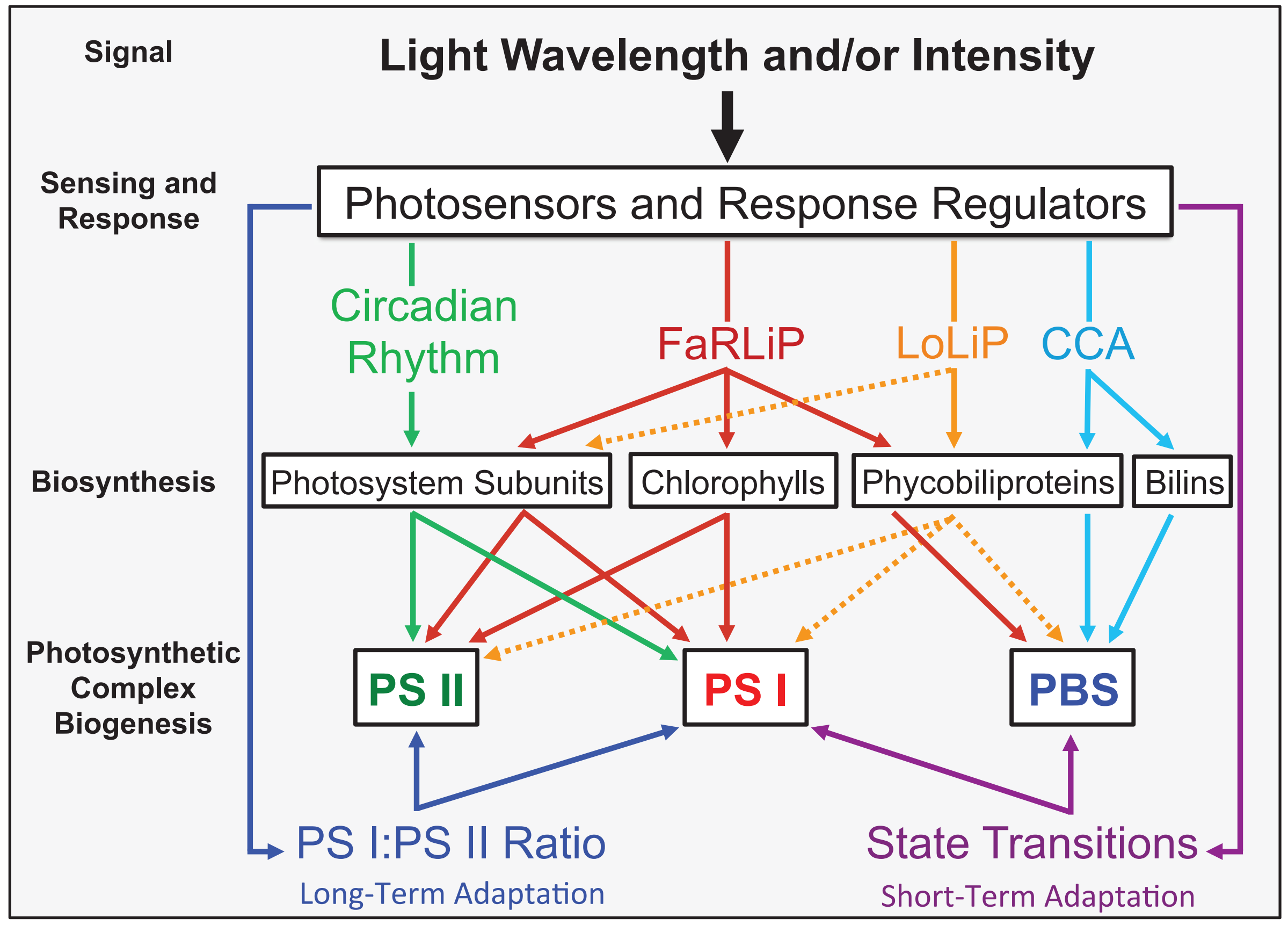


Figure 2
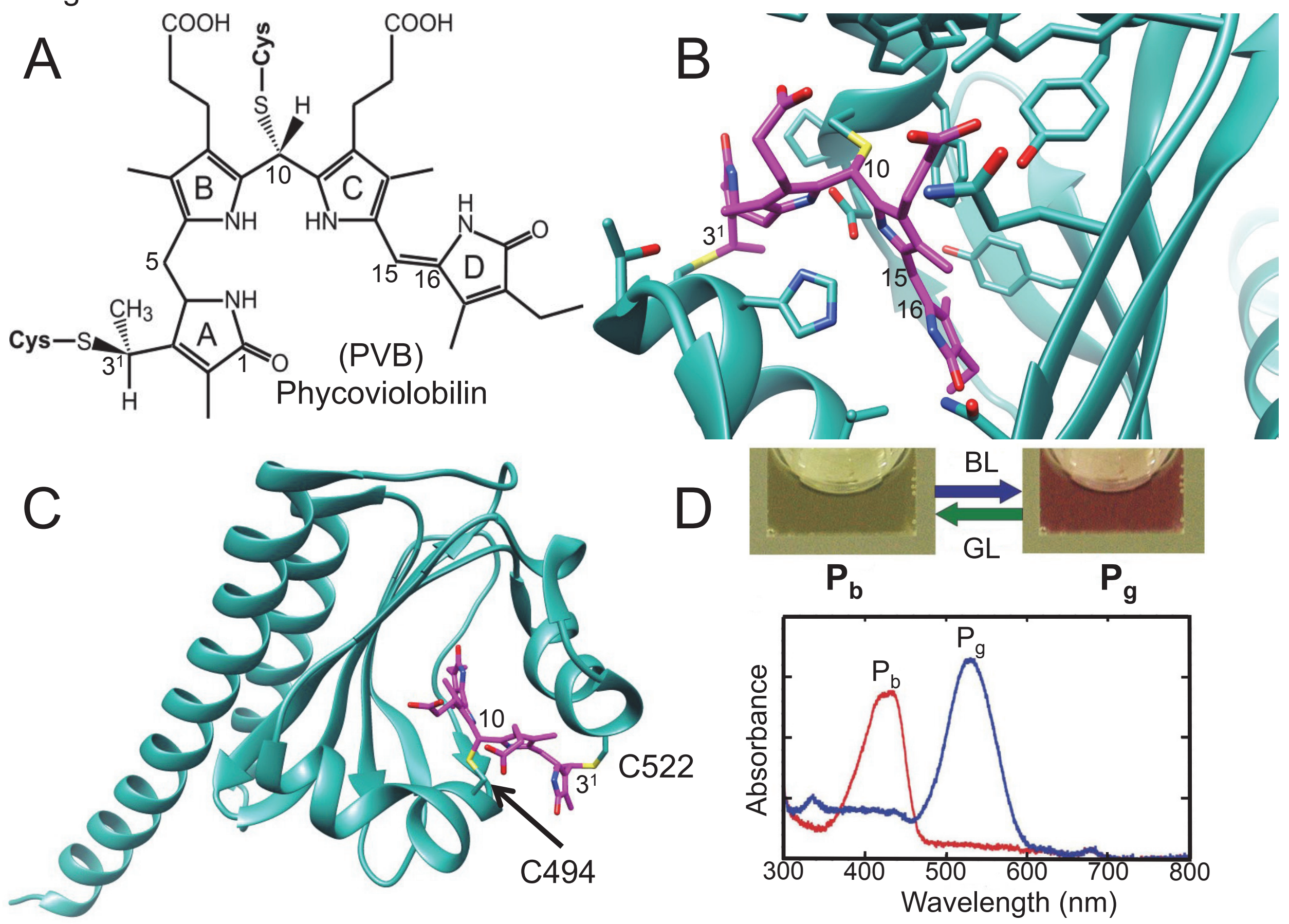
Figure 3
WL
RL
GL
FRL
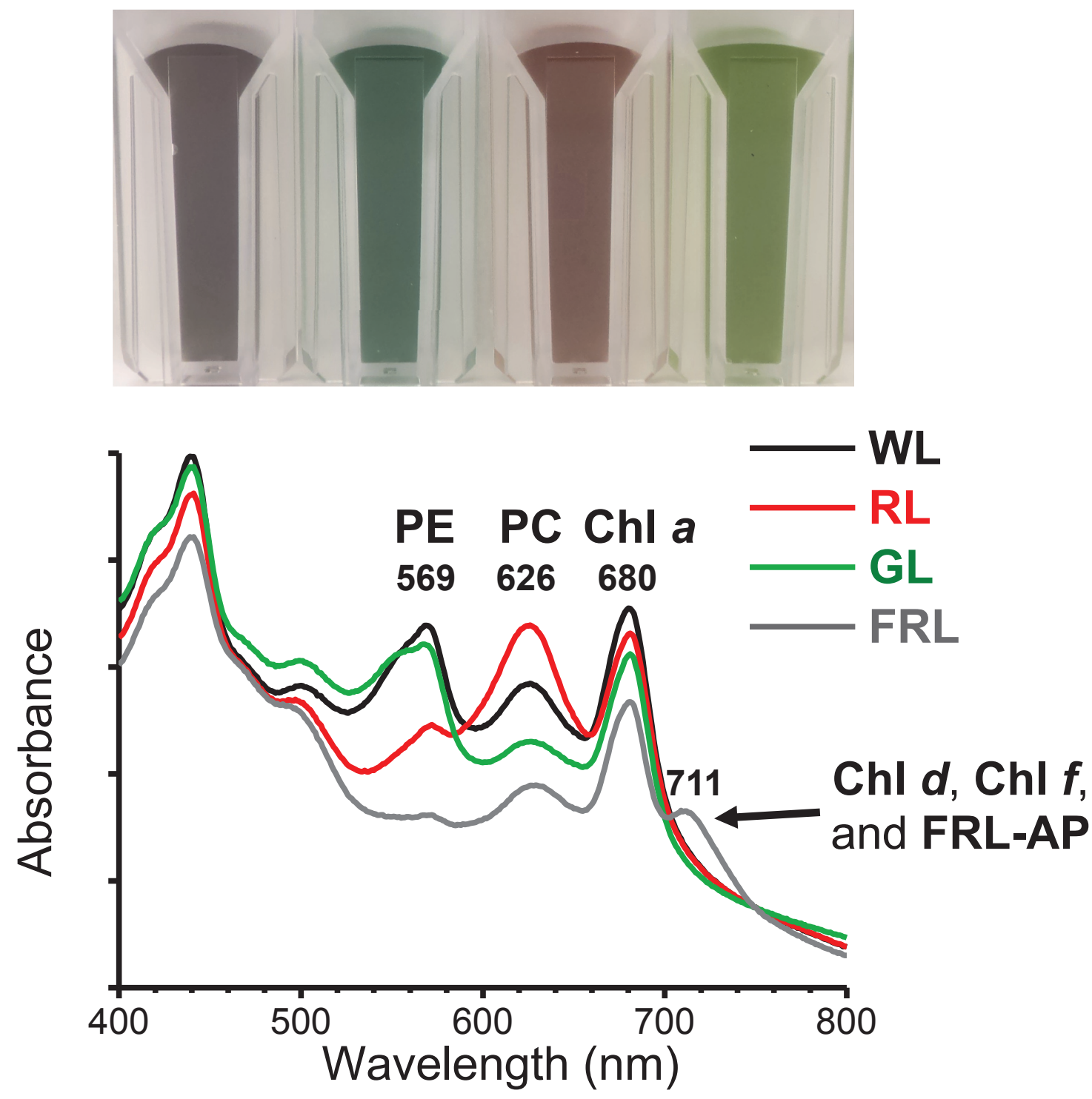
Figure 4
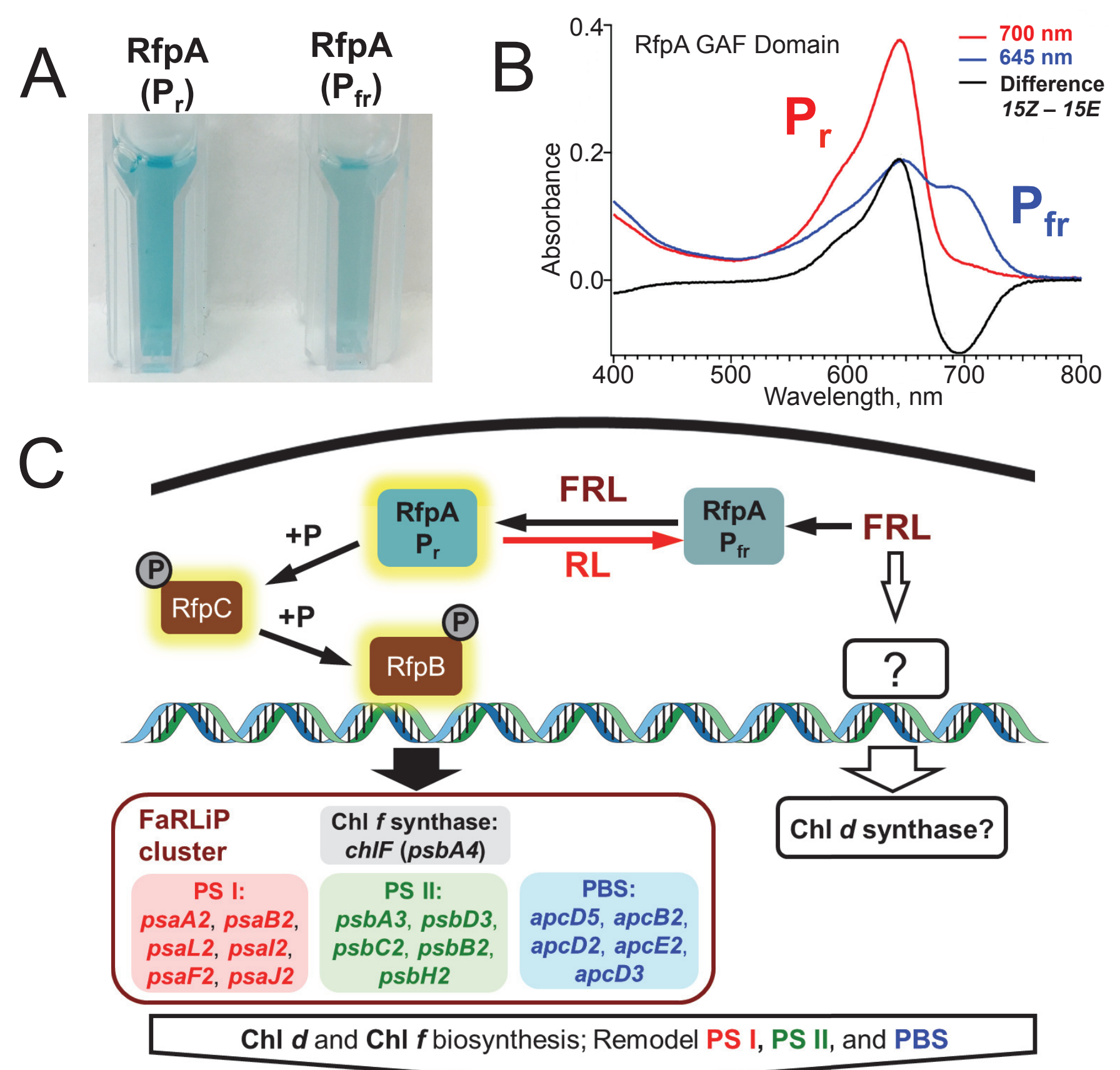

Absorb FRL for growth 